\title{
Encapsulation for efficient electrical injection of photonic crystal defect mode surface-emitting lasers
}

\author{
Weidong Zhou ${ }^{\text {a) }}$ \\ NanoFAB Center, Department of Electrical Engineering, University of Texas at Arlington, \\ Texas 76019-0072
}

(Received 31 August 2005; accepted 13 December 2005; published online 31 January 2006)

\begin{abstract}
We propose and report an encapsulated photonic crystal concept for efficient electrical injection of photonic crystal surface-emitting lasers. Detailed rate equation analysis reveals a significant threshold current reduction by minimizing the surface-state-induced carrier loss. The impact of encapsulation on the photonic band gap is discussed for different encapsulation conditions. Experimental results on encapsulation process are reported, based on nanoparticle self-assembly. (C) 2006 American Institute of Physics. [DOI: 10.1063/1.2171483]
\end{abstract}

Owing to the ability of spontaneous emission control, photonic crystal $(\mathrm{PC})^{1}$ has been the subject of active research for a large variety of light-emitting devices, mostly based on the planar two-dimensional (2D) air-hole photonic crystal slab waveguide (PCS) structures. ${ }^{2,3}$ Enhanced spontaneous emission can be realized in photonic crystal surface-emitting lasers (PCSELs), where lasing is due to the defect mode formed in the center of $2 \mathrm{D}$ photonic crystals with a defect. $^{1,4-6}$ These lasers are most promising in realizing thresholdless lasers and single-photon sources. ${ }^{7}$

Optically pumped PCSELs have been demonstrated and reported. ${ }^{4}$ It was the first experimental proof of surfaceemitting lasing due to spontaneous emission control in photonic crystal defect mode cavities. ${ }^{4,5}$ Shortly thereafter, an electrically injected device was proposed and demonstrated by the author and co-workers, ${ }^{6,8}$ with experimental verification of large spontaneous emission factor $(\beta=0.06)$, a figure of merit for spontaneous emission control, two orders larger than the values in conventional semiconductor lasers and vertival-cavity surface-emitting lasers (VCSELs). Significant progress has been made since then, with various configurations proposed and demonstrated. ${ }^{9-15}$

Depsite the fact that a large spontaneous emission factor has been demonstrated by various groups, ${ }^{8,13,14}$ the lasing threshold is still relatively high $(>250 \mu \mathrm{A})$ for these ultracompact cavity PCSELs, having a photon cavity size less than $(\lambda / n)^{3}$, where $\lambda$ is the emission wavelength and $n$ is the effective refractive index of the slab waveguide. With air holes etched through the whole $p-i-n$ cavity, in order to avoid the possible electrical short-circuit of the laser $p-i-n$ structure, a top metal ring contact has to be placed outside the air-hole region. ${ }^{8,13}$ Such a configuration leads to a major limitation in threshold current reduction in these PCSELs, as will be examined in this letter. We report here the threshold dependence of PCSELs by investigating the impact of spontaneous emission factor $(\beta)$ as well as the surface-stateinduced nonradiative carrier losses $R_{\mathrm{nr}}$. Based on our study, we propose and develop an encapsulation concept for the air-hole based 2D photonic crystal slab structures.

Threshold current calculation based on microcavity rate equation analysis ${ }^{16-18}$ was carried out on air-hole triangular lattice photonic crystal defect mode based surface-emitting

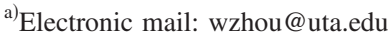

lasers. The carrier density $N_{a}$ in the active region $V_{a}$ and the photon density $N_{P}$ in the photon cavity $V_{P}$ can be described by the following rate equations:

$$
\begin{aligned}
& \frac{d N_{a}}{d t}=\eta_{\text {in }} \frac{I_{a}}{q V_{a}}-\left(\frac{S_{a}}{V_{a}} v_{s} N_{a}+B N_{a}^{2}+C N_{a}^{3}\right)-G\left(N_{a}\right) N_{P}, \\
& \frac{d N_{P}}{d t}=\Gamma G\left(N_{a}\right) N_{P}+\beta B N_{a}^{2}-\frac{N_{P}}{\tau_{P}},
\end{aligned}
$$

where $I_{a}$ is the injection current. The stimulated emission carrier-dependent gain $G\left(N_{a}\right)$ can be expression with threeparameter model as

$$
G\left(N_{a}\right)=v_{g} g\left(N_{a}\right)=\frac{c}{n_{\mathrm{eff}}} \frac{g_{0}}{1+\varepsilon N_{P}} \ln \left(\frac{N_{a}+N_{s}}{N_{0}+N_{s}}\right)
$$

The surface area $S_{a}$ can be related to the total number of air holes $N_{\text {hole }}$ with the following equation: $S_{a}=2 \pi r d_{a} N_{\text {hole }}$, where $r$ is the air-hole radius, and $d_{a}$ is the active region thickness. For simplicity, we assume $N_{\text {hole }}$ to be the number of air holes enclosed inside the metal ring contact. A summary of all the symbols and parameters used for the simulation can be found in Table I.

Calculated threshold currents and the light-current curves (log-log scale plot) are plotted in Figs. 1(a) and 1(b), respectively. The parameters used in our simulation are based on the electrically injected single-defect InP based PCSEL structure and the experimental data reported in Ref. 13 where the top metal ring contact is made outside the PC air column region (top inset of a), assuming a surface recombination velocity of $10^{4} \mathrm{~m} / \mathrm{s}$ for InGaAsP quantum-well (QW) lasers, and a total of seven periods of air holes $\left(\mathrm{N}_{\text {hole }}=168\right)$ enclosed within the metal ring contact region. The minimal threshold is limited to $100 \mu \mathrm{A}$ and above, even for a spontaneous emission factor of unity. On the other hand, if we can reduce the surface recombination impact, by either reducing the surface recombination velocity (through surface passivation) and/or by placing the ring contact close to the center light emission core region, the minimal threshold can approach $10 \mu \mathrm{A}$ or below, where we assume only one period of air holes $\left(N_{\text {hole }}=6\right)$ contributing to the nonradiative surface recombination process, as shown in the bottom inset of Fig. 1(a). Note that the simulation carried out here did not 
TABLE I. Symbols and parameters used in the simulation for InGaAsP QW-based PCSELs.

\begin{tabular}{lccl}
\hline \hline \multicolumn{1}{c}{ Parameters } & Symbol & Unit & Value \\
\hline Wavelength & $\lambda$ & $\mathrm{nm}$ & 1550 \\
Transparent carrier density & $N_{0}$ & $\mathrm{~cm}^{-3}$ & $2 \times 10^{18}$ \\
Offset carrier density & $N_{s}$ & $\mathrm{~cm}^{-3}$ & $1 \times 10^{18}$ \\
Gain compression factor & $\varepsilon$ & $\mathrm{cm}^{3}$ & $1.5 \times 10^{-17}$ \\
Differential gain & $d g / d N$ & $\mathrm{~cm}^{2}$ & $1.0 \times 10^{-15}$ \\
Surface recombination velocity & $v_{s}$ & $\mathrm{~cm}^{3} / \mathrm{s}$ & $1 \times 10^{4}$ \\
Bimolecular & $B$ & $\mathrm{~cm}^{3} / \mathrm{s}$ & $1.2 \times 10^{-10}$ \\
Auger & $C$ & $\mathrm{~cm}^{6} / \mathrm{s}$ & $3.5 \times 10^{-30}$ \\
Aperture size & $A_{a}$ & $\mathrm{~cm}^{2}$ & $1.47 \times 10^{-8}$ \\
Active volume & $V_{a}$ & $\mathrm{~cm}^{3}$ & $1.72 \times 10^{-13}$ \\
Cavity area & $A_{p}$ & & $3 \times 10^{-9}$ \\
Photon cavity volume & $V_{p}$ & $\mathrm{~cm}^{3}$ & $5.87 \times 10^{-14}$ \\
Or cavity $Q$ & & $\cdots$ & 2500 \\
Confinement factor & $\Gamma$ & $\ldots$ & 0.05 \\
Injection efficiency & $\eta_{\text {in }}$ & $\ldots$ & 0.8 \\
Extraction efficiency & $\eta_{\mathrm{o}}$ & $\cdots$ & 0.6 \\
\hline \hline
\end{tabular}

take into account the possible optical losses caused by placing metal ring contact closer to the cavity. This loss may ultimately affect laser threshold and performance, where the cavity is ultrasmall and the threshold is ultralow. Careful optimization of cavity mode control and metal ring contact placement can be done to minimize this loss.

We propose an "encapsulated" photonic crystal (EPC) concept, where an encapsulating cap, is applied on top of the

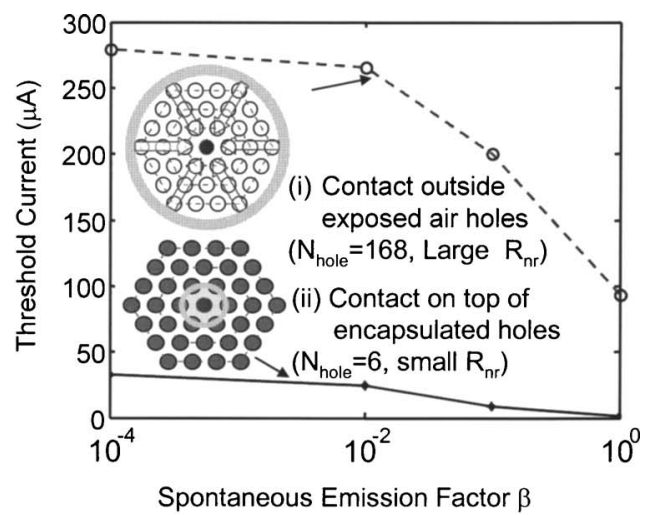

(a)

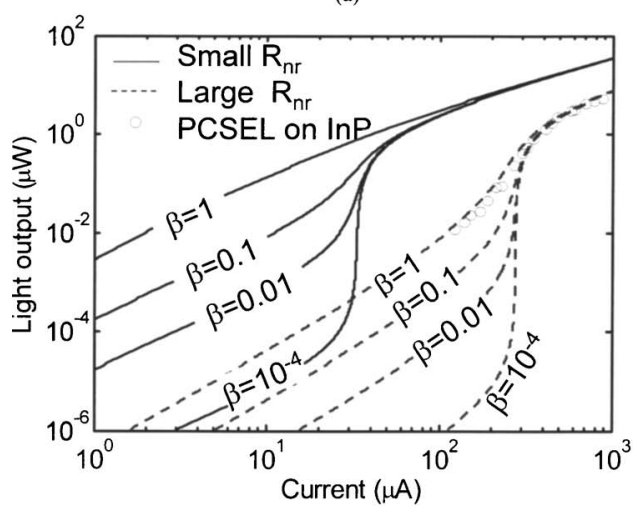

(b)

FIG. 1. Impact of surface recombination on InP based PCSEL thresholds $I_{\mathrm{th}}$ : (a) $I_{\mathrm{th}}$ reduction with beta $(\beta)$ for (i) large nonradiative recombination $R_{\mathrm{nr}}$ (top) and (ii) small $R_{\mathrm{nr}}$ (bottom). (b) Corresponding $L I$ plots fitted with experimental data (PCSEL on InP) from Ref. 13.

Downloaded 24 Sep 2010 to 129.107.76.82. Redistribution subject to AIP license or copyright; see http://apl.aip.org/about/rights_and_permissions

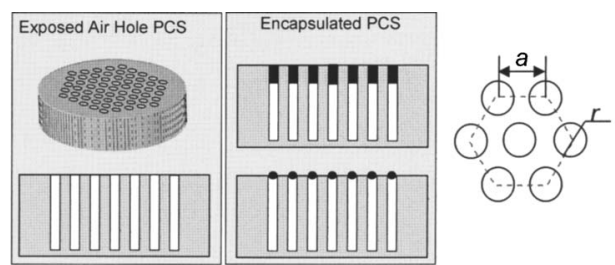

(a)
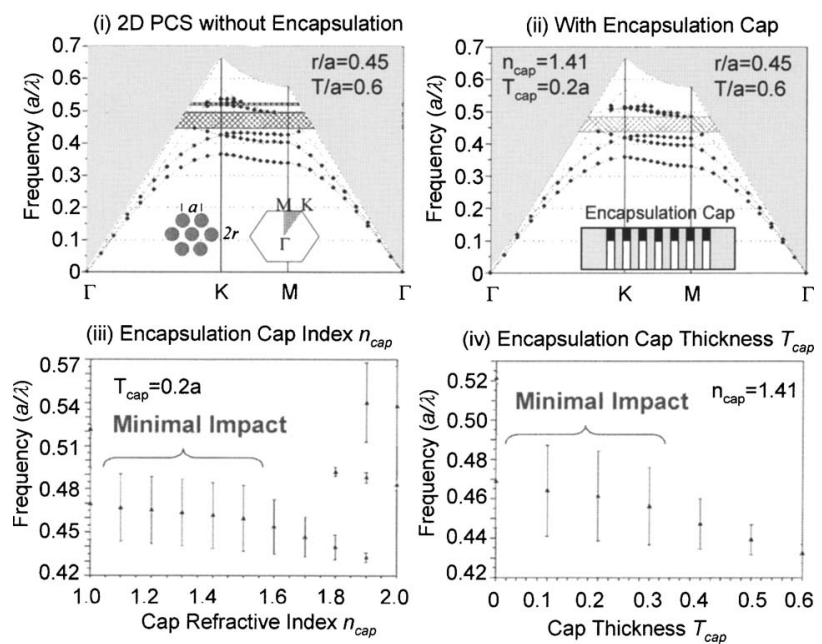

(iv) Encapsulation Cap Thickness $T_{\text {cap }}$

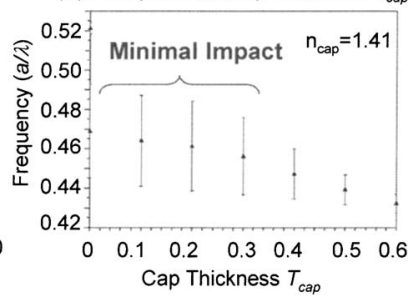

(b)

FIG. 2. (a) Proposed encapsulated photonic crystal slab waveguide with encapsulation cap (top right) or nanoparticle encapsulation (bottom right); (b) Simulated band structures for 2D PCS (i) without and (ii) with encapsulation caps. Assume encapsulation cap refractive index $n_{\text {cap }}=1.41$ and thickness $T_{\text {cap }}=0.2 a$, where $a$ is the lattice period. Photonic band-gap impact due to encapsulation layer (iii) refractive index and (iv) thickness variations. Note large process tolerances.

2D air-hole photonic crystal slab waveguide. A schematic view of the proposed EPC is shown in Fig. 2(a), where an encapsulation cap is on top of the otherwise exposed air holes. The cap enables the efficient electrical injection scheme by placing the metal contact right on top of the air holes near the center of the light emission core. It can also provide protection to the fabricated air-hole structure from any subsequent fabrication processes and enables the fabrication of photonic crystal based photonic integrated circuits, while maintaining the integrity and PC quality.

An air-slab-oxide waveguide structure is used for the simulation, with the simulation parameters summarized in Table II. The simulation was carried out with plane wave expansion technique (RSoft, Inc.). Encapsulation thickness impact (varying $T_{\text {cap }}$ with fixed refractive index $n_{\text {cap }}$ ) and encapsulation material index impact (varying $n_{\text {cap }}$ with fixed

TABLE II. 2D PCS structural parameters used in the simulation.

\begin{tabular}{lccl}
\hline \hline \multicolumn{1}{c}{ Parameters } & Symbol & Unit & Value \\
\hline Lattice constant & $a$ & a & 1 \\
Air-hole radius & $r$ & a & 0.45 \\
Slab waveguide refractive index & $n_{\text {slab }}$ & $\ldots$ & 3.464 \\
Slab waveguide thickness & $T_{\text {slab }}$ & a & 0.6 \\
Substrate refractive index & $n_{\text {sub }}$ & $\ldots$ & 1.414 \\
Substrate thickness & $T_{\text {sub }}$ & a & 100 \\
Encapsulation cap refractive & & & \\
index & $n_{\text {cap }}$ & $\ldots$ & 1 to 2 \\
Encapsulation cap thickness & $T_{\text {cap }}$ & a & 0 to 0.6 \\
\hline \hline
\end{tabular}




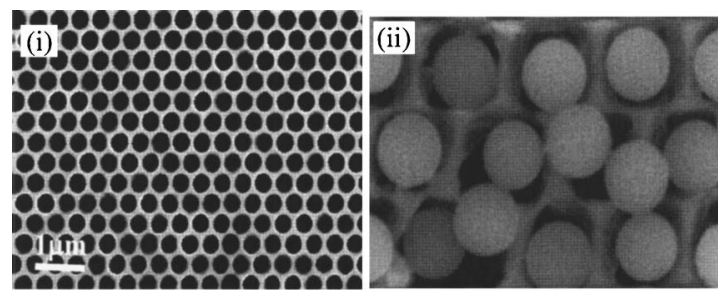

(a)

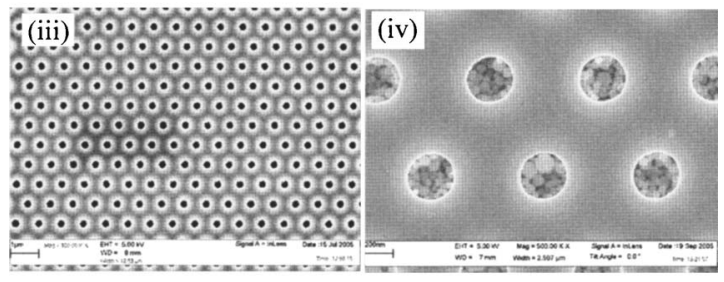

(b)

FIG. 3. (a) 2D PCS SEMs (i) before and (ii) after encapsulation with nanoparticle sizes larger (for encapsulation) than air-hole sizes. (b) 2D PCS SEMs (iii) before and (iv) after encapsulation with nanoparticle sizes much smaller (for backfilling) than air-hole sizes.

$\left.T_{\text {cap }}\right)$ are shown in Fig. 2(b). The simulation results suggest that the impact on photonic band-gap position is minimal for a large range of encapsulation material refractive indexes $(n$ from 1 to 1.6) and thicknesses (up to half-slab waveguide thickness $T$ ). We also found, by carefully choosing the encapsulation material with proper refractive index ( $n_{\text {cap }}$ $=1-1.2,1.5-2.0)$, that it is possible to have a wide band gap in a fully encapsulated structure; that is, the air holes are completely backfilled with the encapsulation material.

Many different approaches can be applied for encapsulation, such as growth, wafer fusion, angled sputtering, spin coating, dip-into-solution, etc. We report here a nanoparticle self-assembly approach based on spin coating. The solutions we use are nanoparticle suspensions. By properly choosing the particle size (the diameter should be slightly larger than air-hole size for encapsulation or smaller than the air-hole size for backfilling) and suspension solution (polar or nonpolar solutions for surface attachment and assembly control), we can spin on the suspension with nanoparticles on top of the air-hole structure. While the suspension solution will fill into the air holes, the nanoparticles will naturally fall onto the top of the air holes. By placing the sample in the oven while slowly spinning the sample, the suspesion liquid will evaporate while the the particles will be left behind, sealing the air holes. Finally, if the sample is cooled down, the vacuum created within the air hole will naturally provide a sealing force for the particles on top.

Experimental results are shown in Fig. 3. Twodimensional PCS on silicon substrate was fabricated with e-beam lithography and reactive ion etching. The air-hole radius is $0.13 \mu \mathrm{m}$. The lattice constants are varied in order to optimize the encapsulation conditions. Scanning electron micrographs (SEMs) of 2D PCS with lattice constant of $0.4 \mu \mathrm{m}$ before and after encapsulation are shown in Figs. 3(a, i) and 3(a, ii), respectively. Polystyrene spheres with nominal size of $300 \mathrm{~nm}$ were spin coated on top of the structure, with spin speed of $3.5 \mathrm{krpm}$ and duration of $40 \mathrm{~s}$. Air dry was applied to the processed samples reported here. Note that nonuniformity is evident in Figure 3(a, ii), due to very high PCS $r / a$ ratio (the ratio between air-hole radius $r$ and lattice constant a). Very high uniformity is achieved in the relative low $r / a$ ratio PCS structures encapsulated with smaller nanoparticles. As shown in Figs. 3(b, iii) and 3(b, iv), silica nanoparticles with nominal sizes of $70 \mathrm{~nm}$ were successfully backfilled into air holes with an air-hole radius of $r=0.18 \mu \mathrm{m}$ and lattice constant of $a=1 \mu \mathrm{m}$. While a low $r / a$ ratio PCS structure favors more uniform assembly and encapsulation, a higher $r / a$ ratio PCS is desirable due to its larger gap size. Further process optimization through weight-volume control of suspension and selective surface property modification is under way in achieving high uniform encapsulation for different structures. Nevertheless, reasonable nonuniformity [Fig. 3(a, ii)] is acceptable since the main purpose of encapsulation is to facilitate the metallization on top of the air-hole region.

In conclusion, we report here an encapsulation concept for widely used planar 2D air-hole PCS waveguide. Theoretical simulation reveals large process tolerance on the encapsulation cap thickness and refractive index. A self-assembly nanoparticle encapsulation process has been developed. Such encapsulated PCs are essential for large-scale multilevel vertical integration and high-performance functional devices, with one specific application to efficient electrical injection of photonic crystal surface-emitting lasers.

The author appreciates the help from Vinay Nair, Geetha Thiruvengadam, Marylene Palard, Shan Tang, and Vance Lee. Work was supported by the State of Texas under a Research Enhancement Grant (REP). Partial support from Air Force Office of Scientific Research under the SPRING program and NSF NNIN facility at University of Texas Austin.

${ }^{1}$ E. Yablonovitch, Phys. Rev. Lett. 58, 2059 (1987).

${ }^{2}$ D. S. Song, S. H. Kim, H. G. Park, C. K. Kim, and Y. H. Lee, Appl. Phys. Lett. 80, 3901 (2002).

${ }^{3}$ S. Noda, M. Yokoyama, M. Imada, A. Chutinan, and M. Mochizuki, Science 295, 1123 (2001).

${ }^{4}$ O. Painter, R. K. Lee, A. Scherer, A. Yariv, J. D. O’Brien, P. D. Daplus, and I. Kim, Science 284, 1819 (1999).

${ }^{5}$ J. Hwang, H. Y. Ryu, D. S. Song, I. Y. Han, H. K. Park, D. H. Jang, and Y. H. Lee, IEEE Photonics Technol. Lett. 12, 1295 (2000).

${ }^{6}$ W. D. Zhou, J. Sabarinathan, B. Kochman, E. Berg, O. Qasaimeh, S. Pang, and P. Bhattacharya, Electron. Lett. 36, 1541 (2000).

${ }^{7}$ C. Santori, D. Fattal, J. Vuckovic, G. S. Solomon, and Y. Yamamoto, Nature (London) 419, 594 (2002).

${ }^{8}$ W. D. Zhou, J. Sabarinathan, P. Bhattacharya, B. Kochman, E. Berg, P. C. Yu, and S. Pang, IEEE J. Quantum Electron. 37, 1153 (2001).

${ }^{9}$ H. Ryu, S. H. Kim, H. G. Park, J. K. Hwang, and Y. H. Lee, Appl. Phys. Lett. 80, 3883 (2002).

${ }^{10}$ M. Loncar, T. Yoshie, and A. Scherer, Appl. Phys. Lett. 81, 2680 (2002).

${ }^{11}$ P.-T. Lee, J. R. Cao, S.-J. Choi, Z. J. Wei, J. D. O'Brien, and P. D. Dapkus, IEEE Photonics Technol. Lett. 14, 435 (2002).

${ }^{12}$ R. Colombelli, K. Srinivasan, M. Troccoli, O. Painter, C. F. Gmachl, D. M. Tennant, A. M. Sergent, D. L. Sivco, A. Y. Cho, and F. Capasso, Science 302, 1374 (2003).

${ }^{13}$ H. Park, S. H. Kim, S. H. Kwon, Y. G. Ju, J. K. Yang, J. H. Baek, S. B. Kim, and Y. H. Lee, Science 305, 1444 (2004).

${ }^{14}$ H. Ryu, M. Notomi, E. Kuramoti, and T. Segawawa, Appl. Phys. Lett. 84, 1067 (2004).

${ }^{15}$ J. Vuckovic, M. Loncar, H. Mabuchi, and A. Scherer, IEEE J. Quantum Electron. 38, 850 (2002).

${ }^{16}$ S. Fan, P. R. Villeneuve, and J. D. Joannopoulos, IEEE J. Quantum Electron. 36, 1123 (2000).

${ }^{17}$ H. Ryu, H. Park, Y. Lee, IEEE J. Sel. Top. Quantum Electron. 8, 891 (2002).

${ }^{18}$ L. A. Coldren and S. W. Corzine, Diode Lasers and Photonic Integrated Circuits (Wiley Interscience, New York, 1995). 